Bull. Austral. Math. Soc.

13D40, 05A15, 13A02, 12H05

VOL. 64 (2001) [291-305]

\title{
ON THE SET OF HILBERT POLYNOMIALS
}

\author{
Alexander B. Levin
}

We characterise the set of all Hilbert polynomials of standard graded algebras over a field and give solutions of some open problems on Hilbert polynomials. In particular, we prove that a chromatic polynomial of a graph is a Hilbert polynomial of some standard graded algebra.

\section{INTRODUCTION}

The purpose of this paper is to describe the set of all Hilbert polynomials of standard graded commutative algebras over a field and to prove some conjectures on Hilbert polynomials.

Throughout the paper $\mathbf{Z}, \mathbf{P}$, and $\mathbf{N}$ denote the sets of integers, positive integers and non-negative integers, respectively.

A polynomial $f(t)$ in one variable $t$ with rational coefficients is called numerical if $f(r) \in \mathbf{Z}$ for all sufficiently large $r \in \mathbf{N}$, that is, there exists $s \in \mathbf{N}$ such that $f(r) \in \mathbf{Z}$ for all $r \in \mathrm{N}, r \geqslant s$.

It is clear that every polynomial with integer coefficients is numerical. As an example of a numerical polynomial with non-integer coefficients one can consider a polynomial $\left(\begin{array}{l}t \\ k\end{array}\right)=t(t-1) \ldots(t-k+1) / k$ ! where $k \in \mathbf{P}$. In what follows, we use some relationships between "binomial" numerical polynomials $\left(\begin{array}{l}t \\ k\end{array}\right)$ that arise from well-known identities for binomial coefficients. In particular, the classical identity $\left(\begin{array}{c}n+1 \\ m\end{array}\right)=\left(\begin{array}{c}n \\ m\end{array}\right)+$ $\left(\begin{array}{c}n \\ m-1\end{array}\right)(n, m \in \mathbf{P}, n \geqslant m)$ implies the polynomial identity $\left(\begin{array}{c}t+1 \\ m\end{array}\right)=\left(\begin{array}{c}t \\ m\end{array}\right)+\left(\begin{array}{c}t \\ m-1\end{array}\right)$ that, in turn, leads to the identity

$$
\sum_{i=0}^{n}\left(\begin{array}{c}
t+i \\
r
\end{array}\right)=\left(\begin{array}{c}
t+n+1 \\
r+1
\end{array}\right)-\left(\begin{array}{c}
t \\
r+1
\end{array}\right)
$$

(As usual, we assume that $\left(\begin{array}{l}t \\ 0\end{array}\right)=1$, and $\left(\begin{array}{l}t \\ k\end{array}\right)=0$ if $k$ is a negative integer.)

It is well-known (see, for example, [7, Proposition 2.1.3]) that any numerical polynomial $f(t)$ of degree $d(d \in \mathbf{N})$ can be represented as

$$
f(t)=\sum_{i=0}^{d} a_{i}\left(\begin{array}{c}
t+i \\
i
\end{array}\right)
$$

Received 8th February, 2001

Copyright Clearance Centre, Inc. Serial-fee code: 0004-9727/01 SA2.00+0.00. 
where $a_{0}, a_{1}, \ldots, a_{d}$ are integers uniquely defined by the polynomial. The coefficient $a_{d}$ is called the leading coefficient of the polynomial $f(t)$, it is denoted by $a_{d}(f)$. The following statement (see [1, Proposition 3.1]) gives another representation of a numerical polynomial.

Proposition 1.1. Let $f(t)$ be a numerical polynomial in one variable $t$ and let $\operatorname{deg} f=d$. Then the polynomial $f(t)$ can be represented in the form

$$
f(t)=\sum_{i=0}^{d}\left[\left(\begin{array}{c}
t+i \\
i+1
\end{array}\right)-\left(\begin{array}{c}
t+i-m_{i} \\
i+1
\end{array}\right)\right]
$$

where $m_{0}, m_{1}, \ldots, m_{d}$ are integers uniquely defined by $f(t)$. Furthermore, the coefficients $a_{i}$ in representation (1.2) of the polynomial $f(t)$ can be expressed in terms of $m_{0}, m_{1}, \ldots, m_{d}$ as follows: $a_{d}=m_{d}$ and

$$
a_{i}=m_{i}+\sum_{j=1}^{d-i}(-1)^{j}\left(\begin{array}{c}
m_{i+j}+1 \\
j+1
\end{array}\right)
$$

for $i=0, \ldots, d-1$.

Let $k$ be a field. By a standard graded $k$-algebra we mean a commutative $k$ algebra $A$ with identity together with a family of its vector $k$-subspaces $\left\{A_{r}\right\}_{r \in \mathrm{N}}$ such that $A=\bigoplus_{r \in \mathbb{N}} A_{r}, A_{0}=k, A_{r} A_{s} \subseteq A_{r+s}$ for any $r, s \in \mathbf{N}$, and $A$ is generated (as a $k$-algebra) by a finite family $\left\{x_{1}, \ldots, x_{n}\right\}$ of elements of $A_{1}$ (so that $A=k\left[x_{1}, \ldots, x_{n}\right]$ ).

The following classical result is due to Hilbert [3].

THEOREM 1.1. With the above notation, there exists a numerical polynomial $\phi_{A}(t)$ in one variable $t$ such that $\phi_{A}(r)=\operatorname{dim}_{k} A_{r}$ for all sufficiently large $r \in \mathbf{N}$ and $\operatorname{deg} \phi_{A} \leqslant n-1$.

The polynomial $\phi_{A}(t)$ whose existence is established by Theorem 1.1 is called the Hilbert polynomial of the standard $k$-algebra $A$. In what follows, the set of all Hilbert polynomials of standard graded algebras is denoted by $\mathcal{H}$ and elements of this set are called Hilbert polynomials. (Thus, $f(t) \in \mathcal{H}$ if and only if there exists a standard graded algebra $A$ over a field such that $f(t)=\phi_{A}(t)$.)

\section{Alternative Descriptions and PROPERTIES of the SET OF HILBERT POLYNOMIALS}

In this section we give several alternative characterisations of the set of Hilbert polynomials of standard graded algebras. First of all note (see, for example, [12, Chapter 7, Section 2, Lemma 1]) that any such polynomial can be treated as a Hilbert 
polynomial of a graded algebra $A=k\left[X_{1}, \ldots, X_{n}\right] / I$ where $k\left[X_{1}, \ldots, X_{n}\right]$ is a polynomial ring in indeterminates $X_{1}, \ldots, X_{n}$ over a field $k$ and $I$ is a homogeneous ideal of this ring. Furthermore, by [2, Chapter 9 , Theorem 12], the set $\mathcal{H}$ coincides with the set of all affine Hilbert polynomials in the sense of the following statement (see [2, Chapter 9, Propositions 3 and 4]).

THEOREM 2.1. Let $J$ be an ideal of a polynomial ring $k\left[X_{1}, \ldots, X_{n}\right]$ over a field $k$ and for any $s \in \mathrm{N}$, let $k\left[X_{1}, \ldots, X_{n}\right]_{\leqslant s}$ denote the set of all polynomials whose total degree does not exceed $s$. Then there exists a numerical polynomial ${ }^{a} \phi(t)$ (called the affine Hilbert polynomial of $J$ ) such that

$$
{ }^{a} \phi(s)=\operatorname{dim}_{k}\left(k\left[X_{1}, \ldots, X_{n}\right] \leqslant s / J \cap k\left[X_{1}, \ldots, X_{n}\right]_{\leqslant s}\right)
$$

for all sufficiently large $s \in \mathbf{N}$ and $\operatorname{deg}^{a} \phi \leqslant n$.

In what follows we consider numerical polynomials associated with subsets of $\mathbf{N}^{n}$ where $n$ is a positive integer. (As usual, elements of such subsets are called $n$-tuples.) The set $\mathbf{N}^{n}$ will be treated as a partially ordered set relative to the product order $\leqslant_{P}$ such that $\left(a_{1}, \ldots, a_{n}\right) \leqslant P\left(b_{1}, \ldots, b_{n}\right)$ if and only if $a_{i} \leqslant b_{i}$ for $i=1, \ldots, n$ ( $\leqslant$ denotes the natural order on $\mathrm{N}$ ). If $A \subseteq \mathbf{N}^{n}$ and $r \in \mathrm{N}$, then $A(r)$ will denote the set of all $n$-tuples $\left(e_{1}, \ldots, e_{n}\right) \in A$ such that $\sum_{i=1}^{n} e_{i} \leqslant r$. Furthermore, $V_{A}$ will denote the set of all $n$-tuples $v=\left(v_{1}, \ldots, v_{n}\right) \in \mathbf{N}^{n}$ that are not greater than or equal to any $n$-tuple from $A$ with respect to the product order $\leqslant p$. (Clearly, an element $v=\left(v_{1}, \ldots, v_{n}\right) \in \mathbf{N}^{n}$ belongs to $V_{A}$ if and only if for any element $\left(a_{1}, \ldots, a_{n}\right) \in A$ there exists $i \in \mathbf{N}, 1 \leqslant i \leqslant n$, such that $a_{i}>v_{i}$.)

The next result is due to Kolchin (see [6, Chapter 0, Lemma 16]).

THEOREM 2.2. Let $A$ be a subset of $\mathbf{N}^{n} \quad(n \in \mathbf{P})$. Then there exists a numerical polynomial $\omega_{A}(t)$ with the following properties.

(i) $\omega_{A}(r)=$ Card $V_{A}(r)$ for all sufficiently large $r \in \mathbf{N}$. (In accordance with our notation, $V_{A}(r)=\left\{\left(x_{1}, \ldots, x_{n}\right) \in V_{A} \mid x_{1}+\cdots+x_{n} \leqslant r\right\}$.)

(ii) $\operatorname{deg} \omega_{A} \leqslant n$.

(iii) $\operatorname{deg} \omega_{A}=n$ if and only if $A=\emptyset$. In this case $\omega_{A}(t)=\left(\begin{array}{c}t+n \\ n\end{array}\right)$.

(iv) $\omega_{A}=0$ if and only if $(0, \ldots, 0) \in A$.

The polynomial $\omega_{A}(t)$, whose existence is established by Theorem 2.2 , is called the Kolchin polynomial of the set $A \subseteq \mathrm{N}^{n}$.

As we have mentioned, a Hilbert polynomial of a standard graded algebra is an affine Hilbert polynomial of an ideal of a polynomial ring, and every affine Hilbert polynomial can be treated as a Hilbert polynomial of a standard graded algebra. Furthermore (see [2, Chapter 9, Propositions 3 and 4]), any polynomial $f(t)$ from the set $\mathcal{H}$ 
can be considered as an affine Hilbert polynomial of some finitely generated monomial ideal $J=\left\langle X_{1}^{e_{11}} \ldots X_{n}^{e_{1 n}}, \ldots, X_{1}^{e_{p 1}} \ldots X_{n}^{e_{p n}}\right\rangle$ of a polynomial ring $k\left[X_{1}, \ldots, X_{n}\right]$ over a field $k\left(p \in \mathbf{P}, e_{i j} \in \mathbf{N}\right.$ for $\left.i=1, \ldots, p ; j=1, \ldots, n\right)$. More precisely, for all $r \in \mathbf{N}$, $f(r)$ is equal to the number of monomials $M=X_{1}^{i_{1}} \ldots X_{n}^{i_{n}}$ such that $\sum_{\nu=1}^{n} i_{\nu} \leqslant r$ and $M$ is not divisible by any $X_{1}^{e_{i 1}} \ldots X_{n}^{e_{i n}}, 1 \leqslant i \leqslant p$ (the canonical images of such monomials $M$ form a basis of the vector $k$-space $\left.k\left[X_{1}, \ldots, X_{n}\right]_{\leqslant r} / J \cap k\left[X_{1}, \ldots, X_{n}\right]_{\leqslant r}\right)$. Thus, if $E$ denotes the finite subset $\left\{\left(e_{11}, \ldots, e_{1 n}\right), \ldots,\left(e_{p 1}, \ldots, e_{p n}\right)\right\}$ of $\mathrm{N}^{n}$, then $f(t)=\omega_{E}(t)$ where $\omega_{E}(t)$ is the Kolchin polynomial of the set $E$. It follows that $\mathcal{H}$ can be treated as the set of all Kolchin polynomials of subsets of $\mathbf{N}^{n}$ (for all $n=1,2, \ldots$ ).

The following two theorems characterise numerical polynomials that belong to the set $\mathcal{H}$. The first of these results is due to Macaulay [10], it describes numerical polynomials that are Hilbert polynomials of standard graded algebras over fields. The second theorem (see [7, Chapter II, Corollary 2.4.8]) was obtained as a characterisation of the set of all Kolchin polynomials of families of $n$-tuples (for $n=1,2, \ldots$ ). As we have seen, both statements concerned with the same set $\mathcal{H}$.

THEOREM 2.3. Let a numerical polynomial $f(t)$ in one variable $t$ be represented in the form (1.3) where $d=\operatorname{deg} f(t)$. Then $f(t) \in \mathcal{H}$ if and only if $m_{0} \geqslant m_{1} \geqslant \ldots \geqslant$ $m_{d} \geqslant 0$.

THEOREM 2.4. Let $f(t)$ be a numerical polynomial in one variable $t, d=$ $\operatorname{deg} f(t)$, and $a_{d}$ the leading coefficient of the polynomial $f(t)$. Then $f(t) \in \mathcal{H}$ if and only if $a_{d}>0$ and the polynomial

$$
f^{*}(t)=f\left(t+a_{d}\right)-\left(\begin{array}{c}
t+d+1+a_{d} \\
d+1
\end{array}\right)+\left(\begin{array}{c}
t+d+1 \\
d+1
\end{array}\right)
$$

belongs to $\mathcal{H}$. (It is easy to see that $\operatorname{deg} f^{*}(t)<d$.)

The next result shows the the set $\mathcal{H}$ is closed under some fundamental operations on polynomials and gives some examples of Hilbert polynomials.

THEOREM 2.5. Let $f(t)$ and $g(t)$ be Hilbert polynomials in one variable $t$, let $m, k \in \mathrm{N}, k>0$, and let $c_{1}, \ldots, c_{k}$ be positive integers. Then the following are Hilbert polynomials:
(i) $f(t)+g(t)$;
(ii) $f(t) g(t)$;
(iii) $f(k t+m)$;
(iv) $k f(t)+m$;
(v) $f(t)-f(t-1)$;
(vi) $\left(\begin{array}{c}t+m \\ m\end{array}\right)$;
(vii) $\left(\begin{array}{c}t+m \\ m\end{array}\right)-\left(\begin{array}{c}t+m-k \\ m\end{array}\right)$; 
(viii) $\quad\left(\begin{array}{c}t+m \\ m\end{array}\right)+\sum_{i=1}^{k} c_{i}\left(\begin{array}{c}t+m-i \\ m\end{array}\right)$.

PROOF: Statements (i)-(v) are independently proven in [1, Theorem 3.5] (where elements of $\mathcal{H}$ are treated as Hilbert polynomials of standard graded algebras) and [7, Section 2.4] (where the results are formulated for Kolchin polynomials). Statements (vi) and (vii) follow from the facts that $\left(\begin{array}{c}t+m \\ m\end{array}\right)$ is the Kolchin polynomial of the empty subset of $\mathbf{N}^{m}$ and $\left(\begin{array}{c}t+m \\ m\end{array}\right)-\left(\begin{array}{c}t+m-k \\ m\end{array}\right)$ is the Kolchin polynomial of the set $\{(k, 0, \ldots, 0)\} \subset \mathbf{N}^{m}$. The last statement is obtained in [7] as a property of the set of Kolchin polynomials (see [7, Lemma 2.4.19]).

REMARK. There are several other descriptions of the set $\mathcal{H}$ that come from differential and difference algebra. In [5] Kolchin introduced a concept of differential dimension polynomial associated with a finitely generated differential field extension. Since then differential dimensional polynomials have played the main role in the dimension theory of differential rings and systems of algebraic differential equations. As shown in [6, Chapter 2, Theorem 6], any differential dimension polynomial can be represented as a finite sum of Kolchin polynomials. This result, together with the first statement of Theorem 2.5, implies that $\mathcal{H}$ coincides with the set of all differential dimension polynomials of finitely generated differential field extensions. Furthermore, as shown in [4], the same set can be considered as the set of all dimension polynomials of finitely generated differential modules over differential fields.

The development of the theory of difference and inversive difference algebraic structures (that is, algebraic structures with the action of sets of injective endomorphisms or automorphisms, respectively) has led to the study of dimension polynomials of difference and inversive difference field extensions. The existence theorems for such polynomials are proven in $[\mathbf{8}, \mathbf{9}]$ and many interesting properties and applications of difference dimension polynomials can be found in [7, Chapters 6-8]. As follows from [7, Proposition 2.5.17, Theorems 6.4.1 and 6.4.8], the set $\mathcal{H}$ can be treated as the set of all dimension polynomials of finitely generated difference field extensions, as well as the set of all characteristic polynomials of finitely generated extensions of inversive difference fields.

\section{Proofs of SOME CONJECTURES ON Hilbert POLYNOMIALS}

In this section we present solutions of some open problems on Hilbert polynomials formulated in [1]. The following two theorems give the main tools for the solutions.

THEOREM 3.1. Suppose that a numerical polynomial $f(t)$ of degree $d$ can be written as

$$
f(t)=\sum_{i=1}^{p} c_{i}\left(\begin{array}{c}
t+d+\alpha_{i} \\
d
\end{array}\right)-\sum_{j=1}^{q} b_{j}\left(\begin{array}{c}
t+d+\beta_{j} \\
d
\end{array}\right)
$$


where $p, q, c_{i}, \alpha_{i}, b_{j}, \beta_{j} \in \mathbf{P}(1 \leqslant i \leqslant p, 1 \leqslant j \leqslant q)$. Furthermore, assume that

$$
\begin{gathered}
a_{d} \geqslant 38 \\
\sum_{i=1}^{p} c_{i} \alpha_{i}>\sum_{j=1}^{q} b_{j} \beta_{j} \\
\max _{1 \leqslant j \leqslant q} \beta_{j} \leqslant 3 \sqrt{a_{d}}
\end{gathered}
$$

and

$$
\sum_{j=1}^{q} b_{j} \leqslant 2 a_{d}
$$

where $a_{d}=a_{d}(f)$ is the leading coefficient of $f(t)$ (clearly, $a_{d}=\sum_{i=1}^{p} c_{i}-\sum_{j=1}^{q} b_{j}$ ).

Then

$$
f^{*}(t)=f\left(t+a_{d}\right)+\left(\begin{array}{c}
t+d+1 \\
d+1
\end{array}\right)-\left(\begin{array}{c}
t+d+1+a_{d} \\
d+1
\end{array}\right)
$$

is a numerical polynomial of degree $d-1$ that can be written as

$$
f^{*}(t)=\sum_{i=1}^{p^{\prime}} c_{i}^{\prime}\left(\begin{array}{c}
t+d-1+\alpha_{i}^{\prime} \\
d-1
\end{array}\right)-\sum_{j=1}^{q^{\prime}} b_{j}^{\prime}\left(\begin{array}{c}
t+d-1+\beta_{j}^{\prime} \\
d-1
\end{array}\right)
$$

where $p^{\prime}, q^{\prime}, c_{i}^{\prime}, \alpha_{i}^{\prime}, b_{j}^{\prime}, \beta_{j}^{\prime} \in \mathbf{P}\left(1 \leqslant i \leqslant p^{\prime}, 1 \leqslant j \leqslant q^{\prime}\right)$ and

$$
\begin{gathered}
a_{d-1}\left(f^{*}\right) \geqslant 38, \\
\sum_{i=1}^{p^{\prime}} c_{i}^{\prime} \alpha_{i}^{\prime}>\sum_{j=1}^{q^{\prime}} b_{j}^{\prime} \beta_{j}^{\prime}, \\
\max _{1 \leqslant j \leqslant q^{\prime}} \beta_{j}^{\prime} \leqslant 3 \sqrt{a_{d-1}\left(f^{*}\right)}, \\
\sum_{j=1}^{q^{\prime}} b_{j}^{\prime} \leqslant 2 a_{d-1}\left(f^{*}\right) .
\end{gathered}
$$


Proof: Let $C=\sum_{i=1}^{p} c_{i}$ and $B=\sum_{j=1}^{q} b_{j}$. Then $a_{d}=C-B$ and

$$
\begin{aligned}
& f^{*}(t)=\sum_{i=1}^{p} c_{i}\left(\begin{array}{c}
t+d+a_{d}+\alpha_{i} \\
d
\end{array}\right)-\sum_{j=1}^{q} b_{j}\left(\begin{array}{c}
t+d+a_{d}+\beta_{j} \\
d
\end{array}\right)+\left(\begin{array}{c}
t+d+1 \\
d+1
\end{array}\right) \\
& -\left(\begin{array}{c}
t+d+1+a_{d} \\
d+1
\end{array}\right) \\
& =\sum_{i=1}^{p} c_{i}\left(\begin{array}{c}
t+d+a_{d}+\alpha_{i} \\
d
\end{array}\right)-\sum_{j=1}^{q} b_{j}\left(\begin{array}{c}
t+d+a_{d}+\beta_{j} \\
d
\end{array}\right)-\sum_{k=1}^{a_{d}}\left(\begin{array}{c}
t+d+k \\
d
\end{array}\right) \\
& =\left[\sum_{i=1}^{p} c_{i}\left(\begin{array}{c}
t+d+a_{d}+\alpha_{i} \\
d
\end{array}\right)-C\left(\begin{array}{c}
t+d+a_{d} \\
d
\end{array}\right)\right]+\left[a_{d}\left(\begin{array}{c}
t+d+a_{d} \\
d
\end{array}\right)\right. \\
& \left.-\sum_{k=1}^{a_{d}}\left(\begin{array}{c}
t+d+k \\
d
\end{array}\right)\right]-\left[\sum_{j=1}^{q} b_{j}\left(\begin{array}{c}
t+d+a_{d}+\beta_{j} \\
d
\end{array}\right)-B\left(\begin{array}{c}
t+d+a_{d} \\
d
\end{array}\right)\right],
\end{aligned}
$$

so that $f^{*}(t)$ can be written as

$$
\begin{aligned}
f^{*}(t)=\sum_{i=1}^{p} c_{i} \sum_{\mu=1}^{\alpha_{i}}\left(\begin{array}{c}
t+d-1+a_{d}+\mu \\
d-1
\end{array}\right)+ & \sum_{k=1}^{a_{d}} \sum_{\nu=1}^{a_{d}-k}\left(\begin{array}{c}
t+d-1+k+\nu \\
d-1
\end{array}\right) \\
& -\sum_{j=1}^{q} b_{j} \sum_{\lambda=1}^{\beta_{j}}\left(\begin{array}{c}
t+d-1+a_{d}+\lambda \\
d-1
\end{array}\right) .
\end{aligned}
$$

Thus, the polynomial $f^{*}(t)$ can be represented in the form (3.6) and

$$
a_{d-1}\left(f^{*}\right)=\sum_{i=1}^{p^{\prime}} c_{i}^{\prime}-\sum_{j=1}^{q^{\prime}} b_{j}^{\prime}=\sum_{i=1}^{p} c_{i} \alpha_{i}+\sum_{k=1}^{a_{d}}\left(a_{d}-k\right)-\sum_{j=1}^{q} b_{j} \beta_{j}
$$

(we apply the notation of formula (3.6) to (3.11)). It follows that

$$
a_{d-1}\left(f^{*}\right)=\left(\sum_{i=1}^{p} c_{i} \alpha_{i}-\sum_{j=1}^{q} b_{j} \beta_{j}\right)+\frac{a_{d}\left(a_{d}-1\right)}{2}
$$

whence $a_{d-1}\left(f^{*}\right)>\left(a_{d}\left(a_{d}-1\right) / 2\right)>a_{d} \geqslant 38$. 
Treating (3.11) as an expression of the form (3.6) we obtain

$$
\begin{aligned}
& \sum_{i=1}^{p^{\prime}} c_{i}^{\prime} \alpha_{i}^{\prime}-\sum_{j=1}^{q^{\prime}} b_{j}^{\prime} \beta_{j}^{\prime} \\
& \quad=\sum_{i=1}^{p} c_{i} \sum_{\mu=1}^{\alpha_{i}}\left(a_{d}+\mu\right)+\sum_{k=1}^{a_{d}} \sum_{\nu=1}^{a_{d}-k}(k+\nu)-\sum_{j=1}^{q} b_{j} \sum_{\lambda=1}^{\beta_{j}}\left(a_{d}+\lambda\right) \\
& \quad=\sum_{i=1}^{p} c_{i}\left(a_{d} \alpha_{i}+\frac{\alpha_{i}\left(\alpha_{i}+1\right)}{2}\right)-\sum_{j=1}^{q} b_{j}\left(a_{d} \beta_{j}+\frac{\beta_{j}\left(\beta_{j}+1\right)}{2}\right)+\sum_{k=1}^{a_{d}} \sum_{\tau=k+1}^{a_{d}} \tau \\
& \quad=a_{d}\left(\sum_{i=1}^{p} c_{i} \alpha_{i}-\sum_{j=1}^{q} b_{j} \beta_{j}\right)+\frac{1}{2}\left[\sum_{i=1}^{p} c_{i} \alpha_{i}^{2}+\sum_{i=1}^{p} c_{i} \alpha_{i}-\sum_{j=1}^{q} b_{j} \beta_{j}^{2}-\sum_{j=1}^{a_{d}} b_{j} \beta_{j}\right]
\end{aligned}
$$

whence

$$
\begin{aligned}
\sum_{i=1}^{p^{\prime}} c_{i}^{\prime} \alpha_{i}^{\prime}-\sum_{j=1}^{q^{\prime}} b_{j}^{\prime} \beta_{j}^{\prime}=\left(a_{d}+\frac{1}{2}\right)\left(\sum_{i=1}^{p} c_{i} \alpha_{i}-\sum_{j=1}^{q} b_{j} \beta_{j}\right) & \\
& +\frac{1}{2}\left[\sum_{i=1}^{p} c_{i} \alpha_{i}^{2}-\sum_{j=1}^{q} b_{j} \beta_{j}^{2}\right]+\frac{a_{d}^{3}-a_{d}}{3}
\end{aligned}
$$

Now conditions (3.2)-(3.5) imply inequality (3.8) for representation (3.11):

$$
\begin{aligned}
\sum_{i=1}^{p^{\prime}} c_{i}^{\prime} \alpha_{i}^{\prime}-\sum_{j=1}^{q^{\prime}} b_{j}^{\prime} \beta_{j}^{\prime} & >-\frac{1}{2} \sum_{j=1}^{q} b_{j} \beta_{j}^{2}+\frac{a_{d}^{3}-a_{d}}{3}>-\frac{1}{2}\left(\sum_{j=1}^{q} b_{j}\right)\left(\max _{1 \leqslant j \leqslant q} \beta_{j}\right)^{2} \\
& >-\frac{1}{2} \cdot 9 a_{d} \cdot 2 a_{d}+\frac{a_{d}^{3}-a_{d}}{3}=\frac{a_{d}\left(a_{d}^{2}-27 a_{d}-1\right)}{3}>0
\end{aligned}
$$

if $a_{d} \geqslant 38$.

Still considering (3.11) as an expression of $f^{*}(t)$ in the form (3.6) we obtain

$$
\max _{1 \leqslant j \leqslant q^{\prime}} \beta_{j}^{\prime}=a_{d}+\max _{1 \leqslant j \leqslant q} \beta_{j}
$$

and

$$
\sum_{j=1}^{q^{\prime}} b_{j}^{\prime}=\sum_{j=1}^{q} b_{j} \beta_{j}
$$


Now the inequality $a_{d-1}\left(f^{*}\right)>\left(a_{d}\left(a_{d}-1\right)\right) / 2$, together with (3.14), (3.4) and the fact that $3 \sqrt{a_{d}\left(a_{d}-1\right) / 2}>a_{d}+3 \sqrt{a_{d}}$ if $a_{d} \geqslant 38$, implies that $\max _{1 \leqslant j \leqslant q^{\prime}} \beta_{j}^{\prime} \leqslant$ $3 \sqrt{a_{d-1}\left(f^{*}\right)}$. Furthermore, equality (3.15) and inequalities (3.4), (3.5) show that

$$
\sum_{j=1}^{q^{\prime}} b_{j}^{\prime} \leqslant\left(\max _{1 \leqslant j \leqslant q} \beta_{j}\right) \sum_{j=1}^{q} b_{j} \leqslant 6 a_{d} \sqrt{a_{d}} .
$$

Since $2 a_{d-1}\left(f^{*}\right)>a_{d}\left(a_{d}-1\right)>6 a_{d} \sqrt{a_{d}}$ if $a_{d} \geqslant 38$, we arrive at inequality (3.10).

As a consequence of Theorem 3.1 we obtain the following statement.

THEOREM 3.2. Suppose that a numerical polynomial $f(t)$ of degree $d$ can be written in the form (3.1) such that $p, q, c_{i}, \alpha_{i}, b_{j}, \beta_{j} \in \mathbf{P}(1 \leqslant i \leqslant p, 1 \leqslant j \leqslant q)$ and conditions (3.2)-(3.5) hold. Then $f(t) \in \mathcal{H}$.

Proof: We proceed by induction on $d$. If $d=0, f(t)=a_{d}>0$, so the statement is obvious.

Let $d>0$ and let the polynomial $f(t)$ be written in the form (3.1) such that $p, q, c_{i}, \alpha_{i}, b_{j}, \beta_{j} \in \mathbf{P}(1 \leqslant i \leqslant p, 1 \leqslant j \leqslant q)$ and conditions (3.2)-(3.5) hold. By Theorem 3.1, inequalities (3.2)-(3.5) imply that the polynomial $f^{*}(t)$ can be written in the form (3.6) and the corresponding inequalities (3.7)-(3.10) hold. By the induction hypothesis, $f^{*}(t) \in \mathcal{H}$ whence $f(t) \in \mathcal{H}$ (see Theorem 2.4).

Theorem 3.2 leads to the following result that solves two conjectures on Hilbert polynomials (see [1, Conjectures 5.3 and 5.4]). In accordance with the notation of [1], for any $d \in \mathbf{N},(t)_{d}$ will denote the polynomial $t(t-1) \ldots(t-d+1)$ in one variable $t$ (in other words, $(t)_{d}=d !\left(\begin{array}{l}t \\ d\end{array}\right)$ ).

THEOREM 3.3. Let $d$ be a positive integer. Then

(i) $3 d\left(\begin{array}{l}t \\ d\end{array}\right) \in \mathcal{H}$.

(ii) If $d \geqslant 4$, then $(t)_{d} \in \mathcal{H}$.

Proof: Since $d$ ! is a multiple of $3 d$ for any $d \in \mathbf{N}, d \geqslant 4$, statement (ii) is a consequence of (i) and Theorem 2.5 (iv). Thus, one needs to prove just the first statement of the theorem.

Let us set $f_{d}(t)=3 d\left(\begin{array}{l}t \\ d\end{array}\right)$ and notice that $f_{i}(t) \in \mathcal{H}$ for $i=1,2,3$, so it is sufficient to prove statement (i) for all $d \in \mathbf{N}, d \geqslant 4$. Indeed, by Theorem 2.5 (vii),

$$
f_{1}(t)=3 t=\left(\begin{array}{c}
t+2 \\
2
\end{array}\right)-\left(\begin{array}{c}
t-1 \\
2
\end{array}\right) \in \mathcal{H}
$$

(it is the Kolchin polynomial of the set $\{(3,0)\} \subset \mathbf{N}^{2}$ ). Furthermore, applying formula (2.1) and Theorem 2.5(i, iv, vi) we obtain

$$
f_{2}^{*}(t)=\left(6\left(\begin{array}{l}
t \\
2
\end{array}\right)\right)^{*}=6\left(\begin{array}{c}
t+6 \\
2
\end{array}\right)-\left(\begin{array}{c}
t+9 \\
3
\end{array}\right)+\left(\begin{array}{c}
t+3 \\
3
\end{array}\right)=3\left(\begin{array}{c}
t+1 \\
1
\end{array}\right)+4\left(\begin{array}{l}
t \\
0
\end{array}\right) \in \mathcal{H}
$$


and

$$
\begin{aligned}
f_{3}^{*}(t)=\left(9\left(\begin{array}{l}
t \\
3
\end{array}\right)\right)^{*} & =9\left(\begin{array}{c}
t+9 \\
3
\end{array}\right)-\left(\begin{array}{c}
t+13 \\
4
\end{array}\right)+\left(\begin{array}{c}
t+4 \\
4
\end{array}\right) \\
& =9\left(\begin{array}{c}
t+2 \\
2
\end{array}\right)+24\left(\begin{array}{c}
t+1 \\
1
\end{array}\right)+9\left(\begin{array}{l}
t \\
0
\end{array}\right) \in \mathcal{H}
\end{aligned}
$$

whence $f_{2}(t) \in \mathcal{H}$ and $f_{3}(t) \in \mathcal{H}$.

Let $d \in \mathbf{N}$ and $d \geqslant 4$. Since the leading coefficient of $f_{d}(t)$ is $3 d$,

$$
f_{d}^{*}(t)=3 d\left(\begin{array}{c}
t+3 d \\
d
\end{array}\right)-\left(\begin{array}{c}
t+4 d+1 \\
d+1
\end{array}\right)+\left(\begin{array}{c}
t+d+1 \\
d+1
\end{array}\right)
$$

Applying identity (1.1) we obtain

$$
\begin{aligned}
f_{d}^{*}(t) & =3 d\left(\begin{array}{c}
t+3 d \\
d
\end{array}\right)-\sum_{i=1}^{3 d}\left(\begin{array}{c}
t+d+i \\
d
\end{array}\right) \\
& =3 d\left[\left(\begin{array}{c}
t+3 d \\
d
\end{array}\right)-\left(\begin{array}{c}
t+d \\
d
\end{array}\right)\right]-\sum_{j=1}^{3 d}\left[\left(\begin{array}{c}
t+d+j \\
d
\end{array}\right)-\left(\begin{array}{c}
t+d \\
d
\end{array}\right)\right] .
\end{aligned}
$$

Using identity (1.1) once again we can write the polynomial $f_{d}^{*}(t)$ as

$$
f_{d}^{*}(t)=3 d \sum_{i=1}^{3 d}\left(\begin{array}{c}
t+d-1+i \\
d-1
\end{array}\right)-\sum_{j=1}^{3 d} \sum_{\nu=1}^{j}\left(\begin{array}{c}
t+d-1+\nu \\
d-1
\end{array}\right)
$$

or

$$
f_{d}^{*}(t)=3 d \sum_{i=1}^{2 d}\left(\begin{array}{c}
t+d-1+i \\
d-1
\end{array}\right)-\sum_{j=1}^{3 d}(3 d-j+1)\left(\begin{array}{c}
t+d-1+j \\
d-1
\end{array}\right)
$$

The last expression can be treated as a representation of the polynomial $f_{d}^{*}(t)$ of degree $d-1$ in the form (3.1) where $p=2 d, c_{i}=3 d, \alpha_{i}=i, q=3 d, b_{j}=3 d-j+1$, and $\beta_{j}=j(1 \leqslant i \leqslant 2 d, 1 \leqslant j \leqslant 3 d)$, so that

$$
\begin{gathered}
a_{d-1}\left(f^{*}\right)=6 d^{2}-\sum_{j=1}^{3 d}(3 d-j+1)=\frac{3 d(d-1)}{2} \\
\sum_{i=1}^{p} c_{i} \alpha_{i}-\sum_{j=1}^{q} b_{j} \beta_{j}=\sum_{i=1}^{2 d} 3 d i-\sum_{j=1}^{3 d}(3 d-j+1) j=\frac{d\left(3 d^{2}-3 d-2\right)}{2} \\
\max _{1 \leqslant j \leqslant q} \beta_{j}=\max _{1 \leqslant j \leqslant 3 d} j=3 d
\end{gathered}
$$


and

$$
\sum_{j=1}^{p} b_{j}=\sum_{j=1}^{3 d}(3 d+1-j)=\frac{3 d(3 d+1)}{2} .
$$

It is easy to see that representation (3.16) of the polynomial $f_{d}^{*}(t)$ does not satisfy conditions (3.2)-(3.5) for all $d \geqslant 4$ (for example, if $d=4$, then $a_{d-1}\left(f_{d}^{*}\right)=18<38$ ). However, the proof of Theorem 3.1 shows that the polynomial $f_{d}^{* *}=\left(f_{d}^{*}\right)^{*}$ of degree $d-2$ can be written as

$$
f_{d}^{* *}(t)=\sum_{i=1}^{p^{\prime}} c_{i}^{\prime}\left(\begin{array}{c}
t+d-2+\alpha_{i}^{\prime} \\
d-2
\end{array}\right)-\sum_{j=1}^{q^{\prime}} b_{j}^{\prime}\left(\begin{array}{c}
t+d-2+\beta_{j}^{\prime} \\
d-2
\end{array}\right)
$$

$\left(p^{\prime}, q^{\prime}, c_{i}^{\prime}, \alpha_{i}^{\prime}, b_{j}^{\prime}, \beta_{j}^{\prime} \in \mathbf{P}\right.$ for $\left.i=1, \ldots, p^{\prime} ; j=1 \ldots, q^{\prime}\right)$ where the parameters $a_{d-2}\left(f^{* *}\right)$

$$
\sum_{i=1}^{p^{\prime}} c_{i}^{\prime} \alpha_{i}^{\prime}-\sum_{j=1}^{q^{\prime}} b_{j}^{\prime} \beta_{j}^{\prime}, \max _{1 \leqslant j \leqslant q^{\prime}} \beta_{j}^{\prime}
$$

and $\sum_{j=1}^{q^{\prime}} b_{j}^{\prime}$ are determined by formulas $(3.12)-(3.15)$ :

$$
\begin{aligned}
a_{d-2}\left(f^{* *}\right) & =\frac{d\left(3 d^{2}-3 d-2\right)}{2}+\frac{1}{2} \cdot \frac{3 d(d-1)}{2}\left[\frac{3 d(d-1)}{2}-1\right] \\
& =\frac{d(3 d+1)\left(3 d^{2}-3 d-2\right)}{8}, \sum_{i=1}^{p^{\prime}} c_{i}^{\prime} \alpha_{i}^{\prime}-\sum_{j=1}^{q^{\prime}} b_{j}^{\prime} \beta_{j}^{\prime} \\
& =\left[\frac{3 d(d-1)}{2}+\frac{1}{2}\right] \frac{d\left(3 d^{2}-3 d-2\right)}{2}+\frac{1}{2}\left[\sum_{i=1}^{2 d} 3 d i^{2}-\sum_{i=1}^{3 d}(3 d-j+1) j^{2}\right] \\
& +\frac{1}{3}\left[\left(\frac{3 d(d-1)}{2}\right)^{3}-\frac{3 d(d-1)}{2}\right] \\
& =\frac{d\left(9 d^{5}-9 d^{4}-4 d^{3}-9 d^{2}-9 d-2\right)}{8}, \\
\max _{1 \leqslant j \leqslant q^{\prime}} \beta_{j}^{\prime} & =\frac{3 d(d-1)}{2}+3 d=\frac{3 d(d+1)}{2},
\end{aligned}
$$

and

$$
\sum_{j=1}^{q^{\prime}} b_{j}^{\prime}=\sum_{j=1}^{3 d}(3 d-j+1) j=\frac{d(3 d+1)(3 d+2)}{2} .
$$


Now it is easy to check that if $d \geqslant 4$, then $a_{d-2}\left(f^{* *}\right) \geqslant 38, \sum_{i=1}^{p^{\prime}} c_{i}^{\prime} \alpha_{i}^{\prime}-\sum_{j=1}^{q^{\prime}} b_{j}^{\prime} \beta_{j}^{\prime}>$ $0, \max _{1 \leqslant j \leqslant q^{\prime}} \beta_{j}^{\prime}<3 \sqrt{a_{d-2}\left(f^{* *}\right)}$ and $\sum_{j=1}^{q^{\prime}} b_{j}^{\prime}<2 a_{d-2}\left(f^{* *}\right)$. By Theorem $3.2, f^{* *}(t) \in \mathcal{H}$. Applying Theorem 2.4, we arrive at the desired inclusion $f_{d}(t)=3 d\left(\begin{array}{l}t \\ d\end{array}\right) \in \mathcal{H}$.

Theorem 3.2 allows us to characterise some other numerical polynomials as Hilbert polynomials of standard graded algebras. The following statement solves two more problems of this type.

\section{THEOREM 3.4. Let $p \in \mathbf{N}, p \geqslant 2$. Then}

(i) $\left(\begin{array}{c}t+p+2 \\ p\end{array}\right)-\left(\begin{array}{c}t+p+3 \\ p-2\end{array}\right) \in \mathcal{H}$;

(ii) $\left(\begin{array}{c}t+p \\ p\end{array}\right)+\left(\begin{array}{c}t+p+1 \\ p\end{array}\right)-\left(\begin{array}{c}t+p+2 \\ p-2\end{array}\right) \in \mathcal{H}$.

Proof: Note first that it is sufficient to prove (i) and (ii) for $p \geqslant 4$, since the inclusion in cases $p=2$ and $p=3$ can be verified directly: setting $f_{p}(t)=\left(\begin{array}{c}t+p+2 \\ p\end{array}\right)-$ $\left(\begin{array}{c}t+p+3 \\ p-2\end{array}\right), \quad g_{p}(t)=\left(\begin{array}{c}t+p \\ p\end{array}\right)+\left(\begin{array}{c}t+p+1 \\ p\end{array}\right)-\left(\begin{array}{c}t+p+2 \\ p-2\end{array}\right)$ and applying Theorem 2.5(viii) we obtain that

$$
\begin{aligned}
f_{2}(t) & =\left(\begin{array}{c}
t+4 \\
2
\end{array}\right)-1=\left(\begin{array}{c}
t+2 \\
2
\end{array}\right)+\left(\begin{array}{c}
t+2 \\
1
\end{array}\right)+\left(\begin{array}{c}
t+3 \\
1
\end{array}\right)-1 \\
& =\left(\begin{array}{c}
t+2 \\
2
\end{array}\right)+2\left(\begin{array}{c}
t+1 \\
1
\end{array}\right)+2\left(\begin{array}{l}
t \\
0
\end{array}\right) \in \mathcal{H} \\
g_{2}(t) & =\left(\begin{array}{c}
t+2 \\
2
\end{array}\right)+\left(\begin{array}{c}
t+3 \\
2
\end{array}\right)-1=2\left(\begin{array}{c}
t+2 \\
2
\end{array}\right)+\left(\begin{array}{c}
t+1 \\
1
\end{array}\right) \in \mathcal{H}
\end{aligned}
$$

and

$$
f_{3}(t)=\left(\begin{array}{c}
t+5 \\
3
\end{array}\right)-\left(\begin{array}{c}
t+6 \\
1
\end{array}\right)=\left(\begin{array}{c}
t+3 \\
3
\end{array}\right)+2\left(\begin{array}{c}
t+2 \\
2
\end{array}\right)+2\left(\begin{array}{c}
t+1-1 \\
1
\end{array}\right)+\left(\begin{array}{l}
t \\
0
\end{array}\right) \in \mathcal{H}
$$

Furthermore, it is easy to check that $g_{3}(t)$ can be written in the form (1.3) as follows:

$$
\begin{aligned}
g_{3}(t)=\left[\left(\begin{array}{c}
t+3 \\
4
\end{array}\right)-\left(\begin{array}{c}
t+3-2 \\
4
\end{array}\right)\right] & +\left[\left(\begin{array}{c}
t+2 \\
3
\end{array}\right)-\left(\begin{array}{c}
t+2-4 \\
3
\end{array}\right)\right] \\
& +\left[\left(\begin{array}{c}
t+1 \\
2
\end{array}\right)-\left(\begin{array}{c}
t+1-9 \\
2
\end{array}\right)\right]+\left[\left(\begin{array}{c}
t \\
1
\end{array}\right)-\left(\begin{array}{c}
t-32 \\
1
\end{array}\right)\right] .
\end{aligned}
$$

Now, Theorem 2.3 shows that $g_{3}(t) \in \mathcal{H}$ (with the notation of Theorem 2.3, we have $d=3$ and $\left.m_{0}=32>m_{1}=9>m_{2}=4>m_{3}=2>0\right)$.

Let $p \geqslant 4$ and let $F_{0}(t)=f_{p}(t)$ and $F_{k}(t)=F_{k-1}^{*}(t)$ for $k=1,2, \ldots$. By Theorem 2.4, if $F_{k}(t) \in \mathcal{H}$ for at least one $k \in \mathbf{N}$, then $f_{p}(t) \in \mathcal{H}$. Direct calculations (with the 
use of formulas (2.1) and (1.1)) show that

$$
\begin{aligned}
F_{4}(t)= & \sum_{i=1}^{23} i\left(\begin{array}{c}
t+p-4+i+1 \\
p-4
\end{array}\right)+23\left(\begin{array}{c}
t+p-4+25 \\
p-4
\end{array}\right)+22\left(\begin{array}{c}
t+p-4+26 \\
p-4
\end{array}\right) \\
& +20\left(\begin{array}{c}
t+p-4+27 \\
p-4
\end{array}\right)+17\left(\begin{array}{c}
t+p-4+28 \\
p-4
\end{array}\right)+13\left(\begin{array}{c}
t+p-4+29 \\
p-4
\end{array}\right) \\
& +8\left(\begin{array}{c}
t+p-4+30 \\
p-4
\end{array}\right)+3\left(\begin{array}{c}
t+p-4+31 \\
p-4
\end{array}\right)-\left(\begin{array}{c}
t+p-4+32 \\
p-4
\end{array}\right) \\
& -3\left(\begin{array}{c}
t+p-4+33 \\
p-4
\end{array}\right)-3\left(\begin{array}{c}
t+p-4+34 \\
p-4
\end{array}\right)-2\left(\begin{array}{c}
t+p-4+35 \\
p-4
\end{array}\right) \\
& -\left(\begin{array}{c}
t+p-4+36 \\
p-4
\end{array}\right) .
\end{aligned}
$$

Considering the last equality as a representation of $F_{4}(t)$ in the form (3.1) (with $d=$ $p-4)$, we see that this representation satisfies conditions (3.2)-(3.5). Indeed, with the notation of Theorem 3.1, we find that

$$
\begin{aligned}
a_{p-4}\left(F_{4}\right) & =372 \geqslant 38, \sum_{i} c_{i} \alpha_{i}-\sum_{j} b_{j} \beta_{j}=7134>0 \\
\max _{j} \beta_{j} & =36<3 \sqrt{a_{p-4}\left(F_{4}\right)}=3 \sqrt{372}
\end{aligned}
$$

and $\sum_{j} b_{j}=10<2 a_{p-4}\left(h_{4}\right)$. By Theorem $3.2, F_{4}(t) \in \mathcal{H}$ whence $f_{p}(t) \in \mathcal{H}$.

Setting $G_{0}(t)=g_{p}(t)$ and $G_{k}(t)=G_{k-1}^{*}(t)$ for $k=1,2, \ldots$, we obtain (after a series of routine manipulations with binomial coefficients) that

$$
\begin{aligned}
G_{4}(t)= & \sum_{i=1}^{23}\left(\begin{array}{c}
t+p-4+i+1 \\
p-4
\end{array}\right)+23\left(\begin{array}{c}
t+p+21 \\
p-4
\end{array}\right)+22\left(\begin{array}{c}
t+p+22 \\
p-4
\end{array}\right)+20\left(\begin{array}{c}
t+p+23 \\
p-4
\end{array}\right) \\
& +17\left(\begin{array}{c}
t+p+24 \\
p-4
\end{array}\right)+13\left(\begin{array}{c}
t+p+25 \\
p-4
\end{array}\right)+8\left(\begin{array}{c}
t+p+26 \\
p-4
\end{array}\right)+3\left(\begin{array}{c}
t+p+27 \\
p-4
\end{array}\right) \\
& -\left(\begin{array}{c}
t+p+28 \\
p-4
\end{array}\right)-3\left(\begin{array}{c}
t+p+29 \\
p-4
\end{array}\right)-3\left(\begin{array}{c}
t+p+30 \\
p-4
\end{array}\right)-2\left(\begin{array}{c}
t+p+31 \\
p-4
\end{array}\right) \\
& -\left(\begin{array}{c}
t+p+32 \\
p-4
\end{array}\right) .
\end{aligned}
$$

Since the parameters of the polynomial $G_{4}(t)$ satisfy conditions (3.2)-(3.5) (with the notation of Theorem 3.1, $d=p-4, a_{d}\left(F_{4}\right)=372, \sum_{i} c_{i} \alpha_{i}=7473, \sum_{j} b_{j} \beta_{j}=$ $339, \max _{j} \beta_{j}=36$, and $\left.\sum_{j} b_{j}=10\right), \quad G_{4}(t) \in \mathcal{H}$ whence $g_{p}(t)$ is a Hilbert polynomial. 
We conclude with the proof of a conjecture on chromatic polynomials of graphs.

Let $G=(V, E)$ be a graph without loops and multiple edges ( $V$ and $E$ denote the set of vertices and the set of edges of the graph $G$, respectively.) Recall that a colouring of a graph is an assignment of a colour to each of the vertices of $G$ in such a way that adjacent vertices have different colours. If the set of all possible colours consists of $k$ elements $(k \in \mathbf{P})$, the colouring is called a $k$-colouring. Thus, one can treat a $k$-colouring as a mapping $\rho: V \rightarrow\{1, \ldots, k\}$ such that $\rho(x) \neq \rho(y)$ if the vertices $x$ and $y$ are adjacent.

It is well-known (see, for example, $[11$, Section 3]) that the number of different $k$-colourings of a graph $G$ is a polynomial function of $k$ with rational coefficients. The corresponding polynomial is denoted by $P_{G}(t)$ and called the chromatic polynomial of the graph $G$. (Thus, the chromatic polynomial of a graph $G$ is a polynomial $P_{G}(t)$ in one variable $t$ with rational coefficients such that for any positive integer $k, P_{G}(k)$ is the number of $k$-colourings of the graph $G$.)

It is easy to see that the chromatic polynomial of a complete graph $K_{n}$ is $(t)_{n}=$ $t(t-1) \ldots(t-n+1)$. Furthermore, as it follows from [11, Theorem 1], a chromatic polynomial of a graph can be represented as a sum of polynomials of the form $(t)_{n}$. This fact, together with Theorem 3.3(ii) and Theorem 2.5(i), implies the following result that solves the conjecture on chromatic polynomials formulated in [1] (see [1, Conjecture $5.2\})$.

THEOREM 3.5. Let $G$ be a graph on at least four vertices (without loops and multiple edges), and let $P_{G}(t)$ be the chromatic polynomial of the graph $G$. Then $P_{G}(t)$ is a Hilbert polynomial.

\section{REFERENCES}

[1] F. Brenti, 'Hilbert polynomials in combinatorics', J. Algebraic Combin. 7 (1998), 127-156.

[2] D. Cox, J. Little and D. O'Shea, Ideals, varieties, and algorithms. An introduction to computational algebraic geometry and commutative algebra (Springer-Verlag, Berlin, Hejdelberg, New York, 1992).

[3] D. Hilbert, 'Über die theorie der algebraischen formen', Math. Annal. 36 (1890), 473-534.

[4] J.L. Johnson, 'Differential dimension polynomials and a fundamental theorem on differential modules', Amer. J. Math. 91 (1969), 239-248.

[5] E.R. Kolchin, 'The notion of dimension in the theory of algebraic differential equations', Bull Amer. Math. Soc. 70 (1964), 570-573.

[6] E.R. Kolchin, Differential algebra and algebraic groups (Academic Press, New York, London, 1973).

[7] M.V. Kondrateva, A.B. Levin, A.V. Mikhalev and E.V. Pankratev, Differential and difference dimension polynomials (Kluwer Academic Publishers, Dordrecht, 1999). 
[8] A.B. Levin, 'Characteristic polynomials of filtered difference modules and of difference field extensions', 'Russian Math. Surveys 33 (1978), 165-166.

[9] A.B. Levin, 'Characteristic polynomials of inversive difference modules and some properties of inversive difference dimension', Russian Math. Surveys 35 (1980), 217-218.

[10] F.S. Macaulay, 'Some properties of enumeration in the theory of modular systems', Proc. London Math. Soc. (2) 26 (1927), 531-555.

[11] R.C. Read, 'An introduction to chromatic polynomials', J. Combin. Theory 4 (1968), $52-71$.

[12] O. Zariski and P. Samuel, Commutative Algebra, Vol. II (D. Van Nostard Co., Inc., London, 1960).

Department of Mathematics

The Catholic University of America

Washington DC 20064

United States of America 